\title{
A Case of Peritoneal Sarcomatoid Mesothelioma with Absence of Occupationl Exposure to Asbestos
}

\author{
Seung Hoon Yoo' ${ }^{1}$, Hee Man Kim², Jea Kun Park³, Mi Sung Kim², Sang Yeop Yi ${ }^{5}$ \\ ${ }^{1}$ Division of Gastroenterology, Department of Internal Medicine, Severance Hospital, Yonsei University College of Med- \\ icine, Seoul, Korea \\ ${ }^{2}$ Division of Gastroenterology, Department of Internal Medicine, Wonju Severance Christian Hospital, Yonsei University \\ Wonju College of Medicine, Wonju, Korea \\ ${ }^{3}$ Department of Surgery, Yeonsei Hangmac Hospital, Goyang, Korea \\ ${ }^{4}$ Department of Radiology, Kangbuk Samsung Hospital, Sungkyunkwan University School of Medicine, Seoul, Korea \\ ${ }^{5}$ Department of Pathology, International St. Mary's Hospital, Catholic Kwandong University College of Medicine, Incheon, \\ Korea
}

\begin{abstract}
Sarcomatoid mesothelioma is not very common, mesothelioma is directly attributable to occupational asbestos exposure, with $90 \%$ of cases showing a history of exposure. A 66-year-old male was admitted with an abdominal pain that persisted for 3 weeks. He had no abdominal mass. Computed tomography showed soft tissue thickening in perihepatic space and nodularities in omentum and peritoneum with ascites. There was no absolute diagnosis evidence in ascites analysis. Although the pathology of ascites was free for malignancy, the patient underwent omentum biopsy for definitive diagnosis. In laproscopic exploration, there was omental cake, peritoneal nodular seeding. It was suspected cancer carcinomatosis. Immunohistochemical findings suggested that it was sarcomatoid masothelioma. This is the rare case of a peritoneal sarcomatoid mesothelioma, without any exposure to asbestos.
\end{abstract}

Key Words: Mesothelioma, Sarcoma

Sarcomatoid mesothelioma is an uncommon type of cancer. This is the most serious of all asbestos-related diseases. It only occurs in 7 to 20 percent of all mesothelioma cases. Idiopathic or spontaneous mesothelioma can also occur in the absence of any exposure to asbestos, with a spontaneous rate in humans of around one per million. We report the rare case of a peritoneal sarcomatoid mesothelioma, without any expo- sure to asbestos. If sarcomatoid mesothelioma cancer's symptoms are cataloged and asbestos exposure history is determined, radiologic imaging studies are to be followed. If radiologic studies show serious abnormality, the acquisition of pathology is needed. Tissue samples are analyzed and examined in the laboratory, which normally contradict or confirm the sarcomatoid mesothelioma. The sarcomatoid mesothelioma's
Corresponding Author: Seung Hoon Yoo, Division of Gastroenterology, Department of Internal Medicine, Yonsei University College of Medicine, 50-1 Yonsei-ro, Seodaemun-gu, Seoul 03722, Korea Tel: +82-2-2228-1930 Fax: +82-2-393-6884 E-mail: shyoo@yuhs.ac

\section{(c) (1) (2)}

Articles published in Kosin Medical Journal are open-access, distributed under the terms of the Creative Commons Attribution Non-Commercial License (http://creativecommons.org/licenses/by-nc/4.0/) which permits unrestricted non-commercial use, distribution, and reproduction in any medium, provided the original work is properly cited.
Received: Jul. 25, 2019

Revised: Oct. 14, 2019

Accepted: Nov. 04, 2019 
tissues are hard to distinguish from healthy tissues in most cases, making it a challenge to accurately diagnose sarcomatoid mesothelioma. ${ }^{1}$ Unfortunately, sarcomatoid mesothelioma cancer is very resistant to treatments. As such mesothelioma has a very poor prognosis. ${ }^{2}$ The average survival of sarcomatoid mesothelioma diagnosed is about 8 months. ${ }^{3}$

\section{CASE}

A 66-year-old male was admitted with a persistent abdominal pain for 3 weeks and dyspepsia. He was a lifelong farmer, taking medication for hypertension and diabetes mellitus. He has lived in one area for lifetime, there was no asbestosrelated facility. He had never been exposed as- bestos, and had received appendectomy, 10 years ago. His smoking status was 20 pack-years, his family history was negative for malignancy. On admission, his vital sign was stable, and direct tenderness was noted over the right side of the abdomen, but mass like lesion was absent. The complete blood count showed a white cell count of $10,800 / \mathrm{mm}^{3}$ (neutrophils $69.6 \%$, lymphocytes $20.2 \%$ ), hemoglobin $14.6 \mathrm{~g} / \mathrm{dL}$, and platelet count of $172,000 / \mathrm{mm}^{3}$. The blood chemistry showed a Total bilirubin $0.7 \mathrm{mg} / \mathrm{dL}$, ALP 67 IU/L, AST/ALT 45/36 U/L, r-GT 87 IU/L, BUN $20.9 \mathrm{mg} / \mathrm{dL}, \mathrm{Cr} 1.0 \mathrm{mg} / \mathrm{dL}$, and CRP $5.30 \mathrm{mg} / \mathrm{dL}$. The CEA was $1.8 \mathrm{ng} / \mathrm{mL}$, and AFP was 11.5 $\mathrm{ng} / \mathrm{mL}$. Blood, urine cultures did not grow any bacteria or fungi. CT showed soft tissue thickening in the perihepatic space and nodularities in omentum and peritoneum with ascites (Fig. 1).

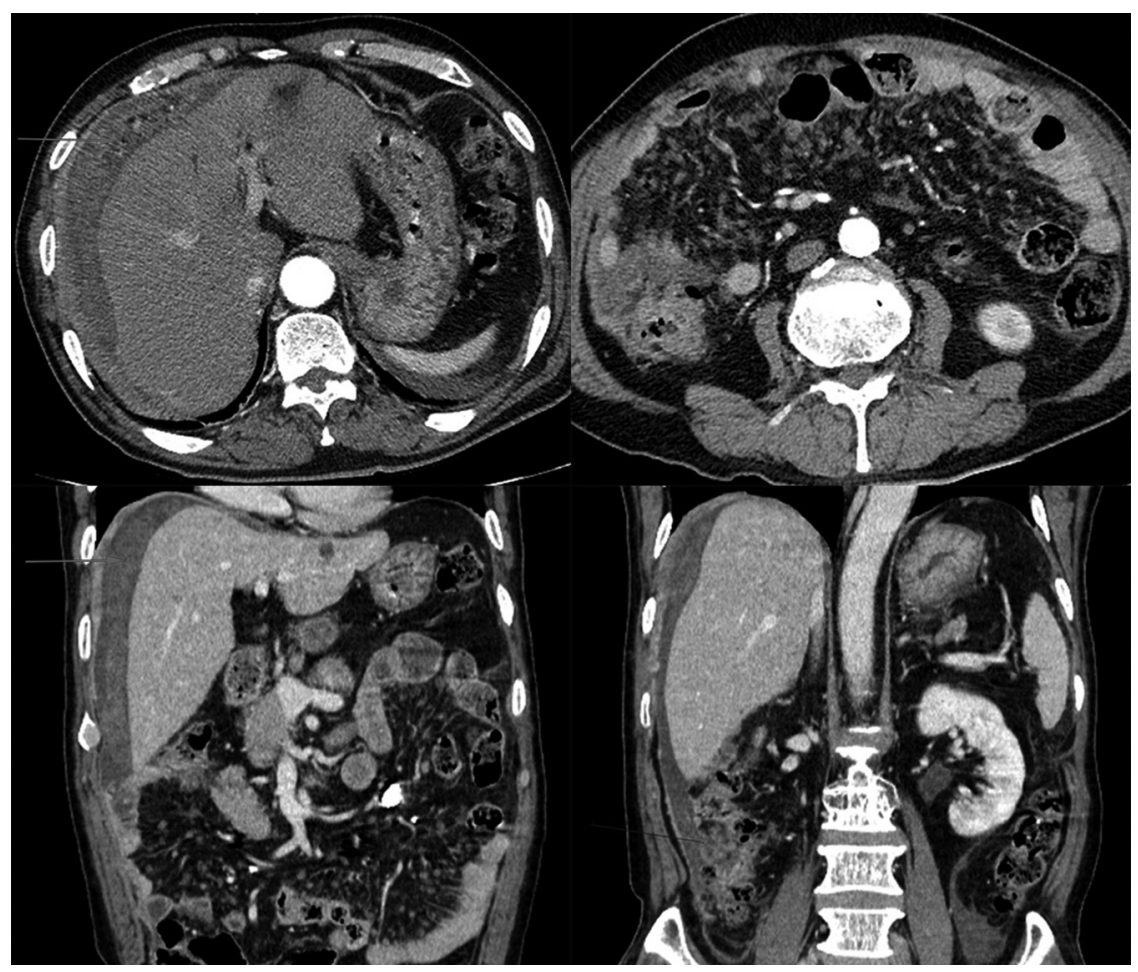

Fig. 1. Computed tomography reveals soft tissue thickening in perihepatic space and peritoneum (thin arrows) with omental nodularities. There are small amounts of ascites in perihepatic space (thick arrows). 
Further, there were multiple calcified lymph nodes in mesentery. Colonoscopy showed sigmoid colon polyp, without mass or inflammation. Histopathology of sigmoid colon polyp showed tubulovilous adenoma with low grade dysplasia. The peritoneal fluid analysis showed $\mathrm{pH}$ 7.0, WBC 5600/uL (lymphocyte 54\%, monocyte 35\%), RBC 5250 /uL, CEA 0.7 $\mathrm{ng} / \mathrm{mL}$, CA 19-9 0.6 U/mL, ADA 28.5 IU/L, INF-gamma (-), and the peritoneal fluid was a exudate (serum-ascites albumin gradient 1.1). Peritoneal fluid cultures did not grow any bacteria or fungi. As a result of the study, we ruled out tuberculous peritonitis or peritoneal carcinomatosis. So we planned diagnostic omentum biopsy, and laproscopic exploration was done. In laproscopic exploration, there was omental cake, peritoneal nodular seeding (Fig. 2). The tumor consists of pleomorphic spindle cells arranged in fascicles or haphazard distribution with focal chronic inflammatory infiltrate. The tumor cells have oval hyperchromatic nuclei and prominent nucleoli. The tumor shows high mitotic activity (67 mitotic figures/50 high power field) and focal necrosis. In immunohistochemical stain, the tumor cells manifest positive reactivity for pancytokeratins, but negative for CD117, CD34, S100 protein, desmin, calretinin, and CEA. According to the histologic findings and immunohistochemical results, the most likely diagnosis is sarcomatoid mesothelioma (Fig. 3). Finally, we concluded to the diagnosis of idio-

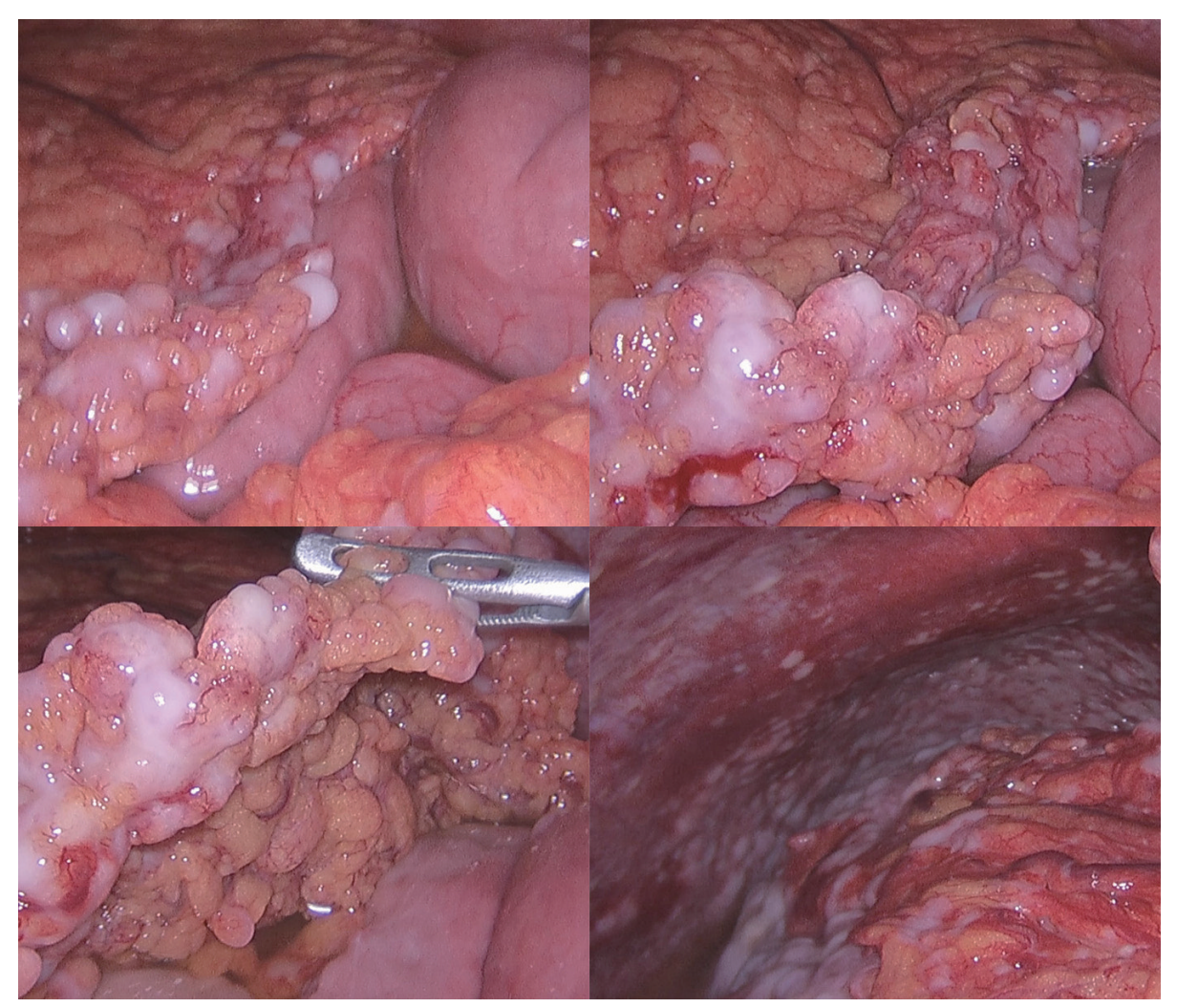

Fig. 2. In laproscopic exploration, there was omental cake, peritoneal nodular seeding . It was suspected cancer carcinomatosis. 


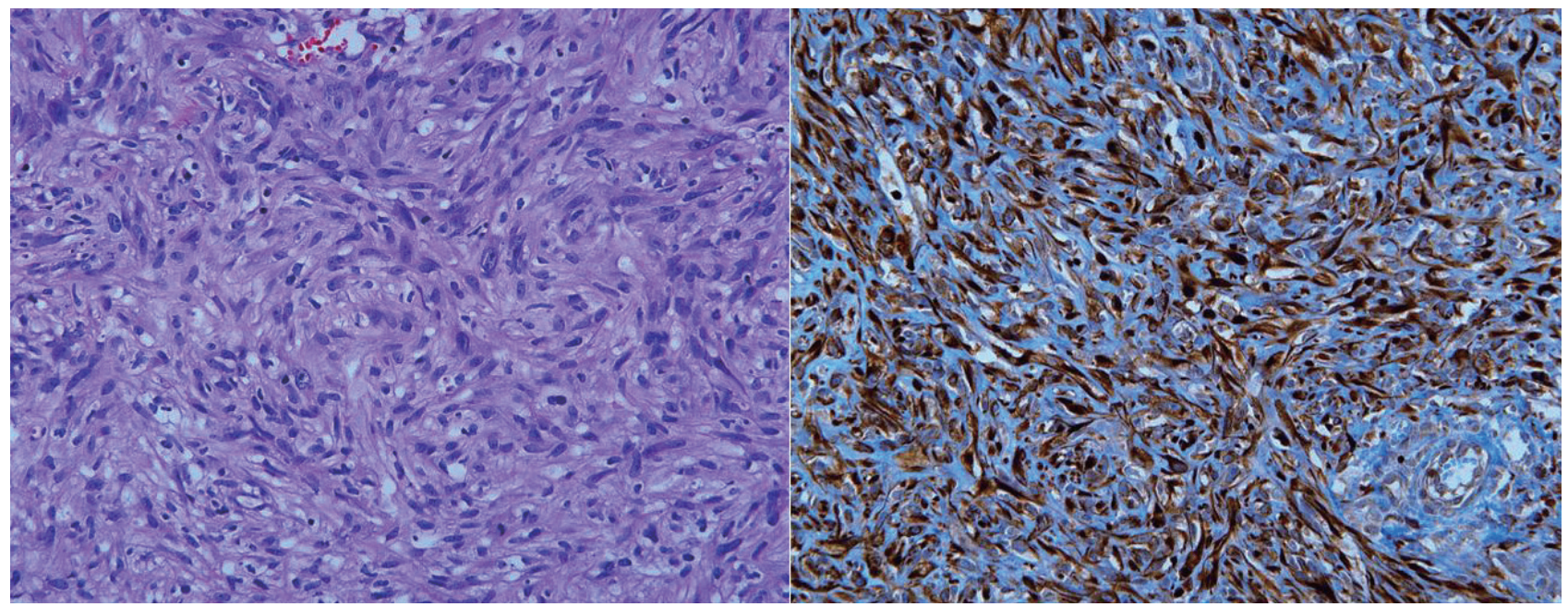

Fig. 3. (Left) Histologically, the tumor consists of pleomorphic spindle cells arranged in fascicles or haphazard distribution, with focal chronic inflammatory infiltrate (H\&E, x 200). (Right) In immunohistochemical stain, the tumor cells manifest positive reactivity for pancytokeratins ( $\mathrm{x} 200)$.

pathic peritoneal sarcomatoid mesothelioma. However, the patient refused management.

\section{DISCUSSION}

Before 1950, malignant mesothelioma was so rare that even its existence was questioned by some pathologists. ${ }^{4}$ However, with the progression of time, the reported cases of malignant mesothelioma increased. Although its use was widely abandoned in the western world in the 1980s, the long latency period between exposure to asbestos and onset of mesothelioma, which can range from 15 to 60 years, meant that the mortality rates from mesothelioma have continued to rise. ${ }^{5}$ Idiopathic or spontaneous mesothelioma can occur in the absence of any exposure to asbestos in both animals and humans, thus, a recent study suggests that idiopathic mesothelioma rate in humans is one per million. ${ }^{6,7}$ In this case, the patient was 66 years without any exposure of asbestos.

Pleural malignant mesothelioma is the most common form of mesothelioma. Typical presenting features are those of chest pain and dyspnea. Breathlessness due to a pleural effusion, without chest pain, has been reported in about $30 \%$ of patients. ${ }^{8}$ Presentation of peritoneal mesothelioma is with non-specific symptoms, which includes the loss of appetite, nausea and vomiting, diarrhea or constipation and occasionally ascites. ${ }^{7}$ Even in this case, there were only non-specific symptoms, including abdominal pain and dyspepsia.

Blood test is not helpful for mesothelioma diagnosis. There can be an elevated erythrocyte sedimentation rate. ${ }^{9}$ The combination of accurate history, examination, radiology, and pathology are essential factors in the diagnosis of mesothelioma. X-ray, CT, MRI and PET have all been used to evaluate the disease. ${ }^{10}$ Malignant 
mesothelioma can be classified as epithelioid, sarcomatoid, or biphasic based on tissue obtained by biopsy. The pathological diagnosis is reached with the aid of immunohistochemistry to demonstrate the presence of mesothelial, epithelial, or true sarcomatous differentiation in the malignant cell. ${ }^{11}$ There is no individual immunohistochemical mesothelial marker that provides $100 \%$ specificity and high sensitivity for the diagnosis of malignant mesothelioma. ${ }^{11}$ Markers, such as keratin, mesothelin, caliretinin, and thorombomodulin have currently been used for the diagnosis and differentiation of mesothelioma. In this case, the tumor consisted of pleomorphic spindle cells arranged in fascicles or haphazard distribution, the tumor cells manifested positive reactivity for pancytokeratins.

Unfortunately, sarcomatoid mesothelioma is resistant to treatment, which means that any treatments, including surgery, chemotherapy, and radiotherapy becomes insignificant in treating sarcomatoid mesothelioma. ${ }^{2}$ The role of surgery is still uncertain, the use of chemotherapy aims to lengthen survival, improve quality of life and provide symptomatic relief. ${ }^{12,13}$ Currently, there is no single drug or combination therapy that could be considered as the standard treatment. ${ }^{14}$ Radiotherapy occasionally induces regression of disease, but there is no evidence that radiotherapy affects survival. ${ }^{12}$ However, it has been helpful in the palliation of pain. ${ }^{15}$ The response to this treatment leads to a poorer prognosis for patients diagnosed with this subtype, as well as a reduced survival time. In this case, we could not confirm treatment outcome because the patient refused management, follow-up.

Life expectancy in sarcomatoid mesothelioma is poor, with median survival varying between 7 and 9 months. ${ }^{3}$ Furthermore, the prognosis of peritoneal mesothelioma is worse than pleural mesothelioma, with a mean survival time of about 7 months. ${ }^{9}$

In Korea, sarcomatoid mesothelioma is very rare. It has a poor prognosis and short survival with any treatment. We report a peritoneal sarcomatoid mesothelioma patient who had never been exposed asbestos.

\section{REFERENCES}

1. Klebe S, Brownlee NA, Mahar A, Burchette JL, Sporn TA, Vollmer RT, et al. Sarcomatoid mesothelioma. Mod Pathol 2010;23:470-9.

2. LoggieBW. Malignant Peritoneal Mesothelioma. Curr Treat Options Oncol 2001;2:395-9.

3. Damhuis RA, Schroten C, Burgers JA. Population-based survival for malignant mesothelioma after introduction of novel chemotherapy. Eur Respir J 2012;40:185-9.

4. Carbone M, Bedrossian CW. The pathogenesis of mesothelioma. Seminars in Diagnostic Pathology 2006;23:56-60.

5. Bianchi C, Giarelli L, Grandi G, Brollo A, Ramani L, Zuch C. Latency periods in asbestos-related mesothelioma of the pleura. Eur J Cancer Prev 1997;6:162-6.

6. Yamate J, Tomita A, Kuwamura M, Mitsunaga F, Nakamura S. Spontaneous peritoneal malignant mesothelioma in a geriatric 
japanese macaque. Exp Anim J 2007;56:155-9.

7. Marchevsky AM, Wick MR. Current controversies regarding the role of asbestos exposure in the causation of malignant mesothelioma. Ann Diagn Pathol 2003;7:321-32.

8. Yates DH, Corrin B, Stidolph PN, Browne K. Malignant mesothelioma in south east England. Thorax 1997;52:507-12.

9. Elmes PC, Simpson JC. The clinical aspects of mesothelioma. Q J Med 1976;45:427-49.

10. Wang ZJ, Reddy GP, Gotway MB, Higgins CB, Jablons DM, Ramaswamy M, et al. Malignant pleural mesothelioma:evaluation with CT, MR imaging, and PET. Radiographics 2004;24:105-19.

11. Marchevsky AM. Application of immunohistochemistry to the diagnosis of malignant mesothelioma. Arch Pathol Lab Med 2008;132:397-401.
12. Davis SR, Tan L, Ball DL. Radiotherapy in the treatment of malignant mesothelioma of the pleura, with special reference to its use in palliation. Australasian Radiol 1994;38:212-4.

13. Berghmans T, Paesmans M, Lalami Y, Louviaux I, Luce S, Mascaux C, et al. Activity of chemotherapy and immunotherapy on malignant mesothelioma. Lung Cancer 2002;38:111-21.

14. van Meerbeeck JP, Gaafar R, Manegold C, van Klaveren RJ, van Marck EA, Vincent M, et al. Randomized phase III study of cisplatin with or without raltitrexed in patients with malignant pleural mesothelioma. Journal of Clinical Oncology 2005;23:6881-9.

15. Ceresoli GL, Gridelli C, Santoro A. Multidisciplinary treatment of malignant pleural mesothelioma. The Oncologist 2007;12:85063. 